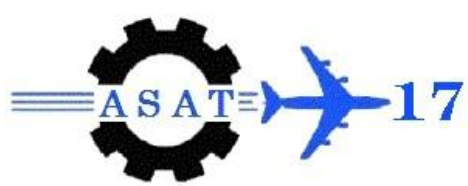

\title{
Modern Signal Processing Techniques for GPR Applications
}

\author{
\{M.A.Mostafa, Fathy M.Ahmed, and Aly S. Attallah\}*
}

\begin{abstract}
This paper introduces a survey of different modern signal processing techniques used in the ground penetrating radar (GPR) for an ongoing research regarding buried objects detection. Detection techniques require extremely high detection rates. In GPR, microwaves signals are transmitted until an object reflects them back, then the reflected signals are processed in order to extract information about the target. This paper discusses different techniques that provide a solution to the main problem of GPR application, which is the strong reflection from unwanted targets.
\end{abstract}

Keywords: GPR; Buried objects; image processing removal; signal processing.

\section{Introduction}

The Ground Penetrating Radar (GPR) approach is one of the most talented technologies for detection and identification of buried objects. The GPR approach uses the difference in the permittivity of both the object and the surrounding medium to detect the target. The difficulty for the GPR system design to identify the buried object depends on three issues. Firstly, the location of the target with respect to the surface of the ground, the target geometrical dimensions (in case of small objects), and irregular shape, and finally how close the object permittivity is to that of the ground. Figure (1) shows the basic scanning operation of the GPR for a region that contains buried objects. In all cases the reflected signal of the target is very weak and overlapped with noise, making it difficult to be distinguished between both without proper signal processing.

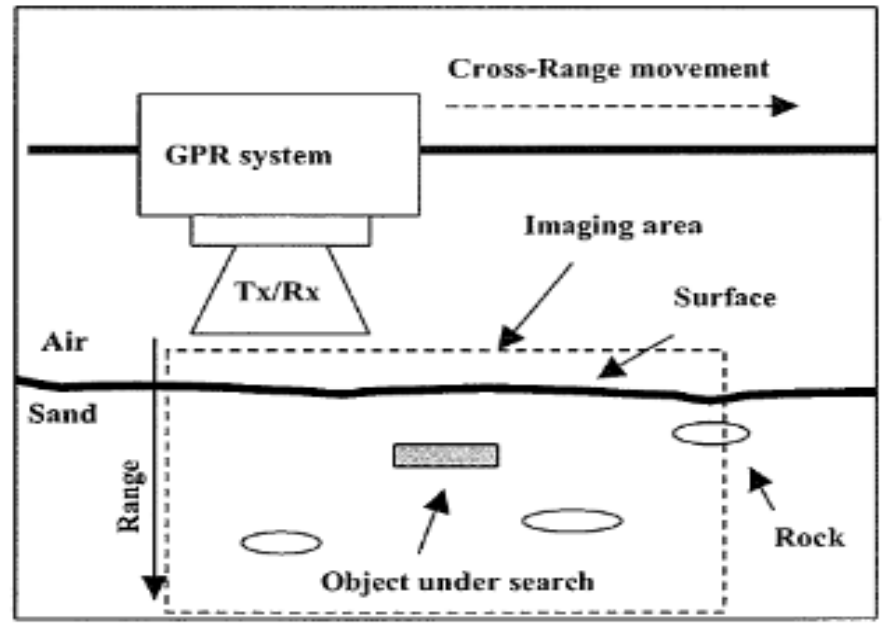

Fig. (1) GPR scanning a sand region that contains buried objects

\footnotetext{
Egyptian Armed Forces, Egypt.
} 
Data that are received from GPR system consist of the following signals:

1. Coupling induced by transmitting and receiving antennas.

2. Reflection by ground surface.

3. Interference either from GPR system itself or from environment.

4. Dispersion or reflection by the underground inhomogeneity.

5 . Reflection by subsurface targets.

Usually, the signals (1) and (2) are called "clutter", which are much more intense than the buried-target reflection due to short distance from transmitting to receiving antenna and the attenuation of the target reflection by underground medium like soil, etc. It is therefore necessary to deploy proper clutter reduction method to be able to detect and identify the target. The signals in (3) and (4) are other interference signals that must be cancelled by using proper signal processing algorithm to avoid affecting the received signals (5) which contains useful information about the underground objects.

In this study, a survey of different modern signal processing techniques developed in GPR is introduced. In GPR, the received microwave signals is processed in order to generate images which contain the responses of objects buried underground. When using GPR, some aspects have to be considered:

1. The reflections from the surface are significantly stronger than those from buried objects.

1- The value of dielectric constants causes small reflection coefficients, hence, producing a weak response which is obscured by the responses of the surface and other objects.

This paper is organized as follows; section 2 summarizes the main signal processing techniques that are used to overcome the main problems of GPR. Conclusion comes at the end of this paper.

\section{The Modern Signal Processing Techniques}

Different techniques that are commonly used so far to generate GPR images considering the problems cited above is introduced briefly in the following sections.

\subsection{Radial Transform and Multi-layer Perceptrons}

The angular radial transform (ART) is a technique used to extract the discriminatory information inherent in the returning signals. ART is a region-based shape descriptor which gives a compact and efficient way of understanding how pixels are distributed in the image and it is used in image indexing and retrieval, human face detection and many other areas. After extracting distinctive information from GPR data, multi-layer perceptrons (MLP) is used with backpropagation algorithm and k-nearest neighbor (k-NN) algorithm to classify the targets, which are the most popular supervised learning approaches [1]. Main advantage of multi-layer perceptrons is that they are quite robust to noise. Even when erroneous samples are present in the dataset, the network can work around such noisy data. However, multi-layer perceptrons are quite obscure to human interpretation.

\subsection{The Neural Detection Technique}

The artificial neural network (ANN) technique gives a promising approach to a more systematic and autonomous detection system. This technique used mainly for pipe detection but can be used for other buried objects [2]. An automatic buried pipe detection algorithm using a twostep ANN scheme on GPR data is used and the detection performance of each ANN in the presence of different signal-to-noise and signal-to- clutter ratios is measured and evaluated. This technique provides a useful way of discriminating an object response from a subsurface layer response. 


\subsection{Polarimetric Analysis Technique}

Although fully polarimetric analysis techniques have been applied to remote sensing radar for charactering different surface scattering properties, using these techniques have not yet been widely adopted for Ground Penetrating Radar (GPR) applications. In 2015, the H-alpha decomposition technique has been applied to polarimetric GPR data for classifying buried metallic targets such as wire branches, a sphere, a plate, and a dihedral [3]. The H-alpha decomposition can separate scatters into four different types according to different depolarization effects: linear polarization, random depolarization (branches, roots, random media), no depolarization (sphere, plates, and horizontal layers), and 90 degrees depolarization (corner reflectors). The $\mathrm{H}$-alpha decomposition method can be utilized in clutter reduction and linear target detection during the preprocessing stage, so, the automatic linear target detection can be achieved in poor signal-to-clutter ratio (SCR) and signal-to-noise ratio (SNR) environments.

\subsection{Time-Frequency Analysis and Waveform Norms}

Recently, various time-frequency analyses has been proposed to discriminate buried land mines from other clutter objects and thus reduce GPR false alarm rates. This approach uses the ChoiWilliams time frequency transform to analyze ultra-wideband signal returns from a range of shallow buried objects [4]. Single Value Decomposition is performed on isolated object timefrequency signatures. The signatures are evaluated using a set of waveform norms that discriminate in time, frequency and energy content. The results obtained from this approach indicate that it could improve land mine detection rates and reduce false alarms.

Typically land mine discrimination, i.e. detection and possibly identification, is achieved using synthetic aperture processing (SAR) techniques and image processing algorithms, such approaches are computationally intensive and require a trained operator. Detection of nonmetallic (NM) and minimum metal (MM) anti-personnel land mines (APLs) with ground penetrating radar (GPR) is often made difficult because of the clutter environment within the first $5 \mathrm{cms}$ of soil surface. On the other hand most APLs are placed within that region to ensure reliable detonation when trod on. The benefits of representing wideband radar data in the timefrequency domain has been recognized and applied in various ways for the purpose of target signature identification. Capturing the scattering properties of a target such as resonances, dispersive propagation, creeping waves and broadband scatterers, results in a signal that has greater information content than the relatively low resolution product obtained from SAR imagery.

\subsection{Forward Looking GPR with Synthetic aperture Antenna}

The problem of detection of explosive hazards is a very important problem all over the world, as these hazards are responsible for uncountable deaths and injuries. To improve the operators save at improvised explosive device (IED) detection, one can utilize so called vehicle mounted forward-looking ground penetrating radar (FLGPR). FLGPR uses an antenna array that directs electromagnetic energy forward, at some incident angle [5]. This technique illuminates an area of ground at some location from the antenna array. While most such GPR systems use time domain information coming from ultra-wide band impulse radar systems, however, scientists suggests a processing technique that analyzes frequency domain data as well. Their results suggest that frequency domain results can distinguish a target from surrounding clutter. They found that, use of a $0.8-1.35 \mathrm{GHz}$ bandwidth radar frequency range produces the lowest false alarm rate of 0.8-2.8 bandwidths analyzed. The imaging system makes use of an implementation of the aperture synthesis scheme in which a single subarray, consisting of one transmitter and one receiver, is used. The full transmit and receive array is realized by moving this subarray to different locations forming the array aperture. 


\subsection{GPR data Normalizing and Background Reduction Techniques}

To visualize GPR data, it requires implementing algorithms to read $3 \mathrm{dr}$ logged file and to plot C-scan and B-scan so that it helps to analyses the complex 3D GPR data in 2D Images and then normalizing the data followed by background removal processing \& feature extracting algorithm. The proposed overall procedure is shown in the figure (2) below. It consists following processes: reading and normalizing the data followed by background removal methods, estimating, noise reduction and visual identification. For the noise reduction, mean subtraction method \& median filter can be used. Number of objects in the data is estimated using Gaussian filter and Image smoothing function applied to reduce the noise.

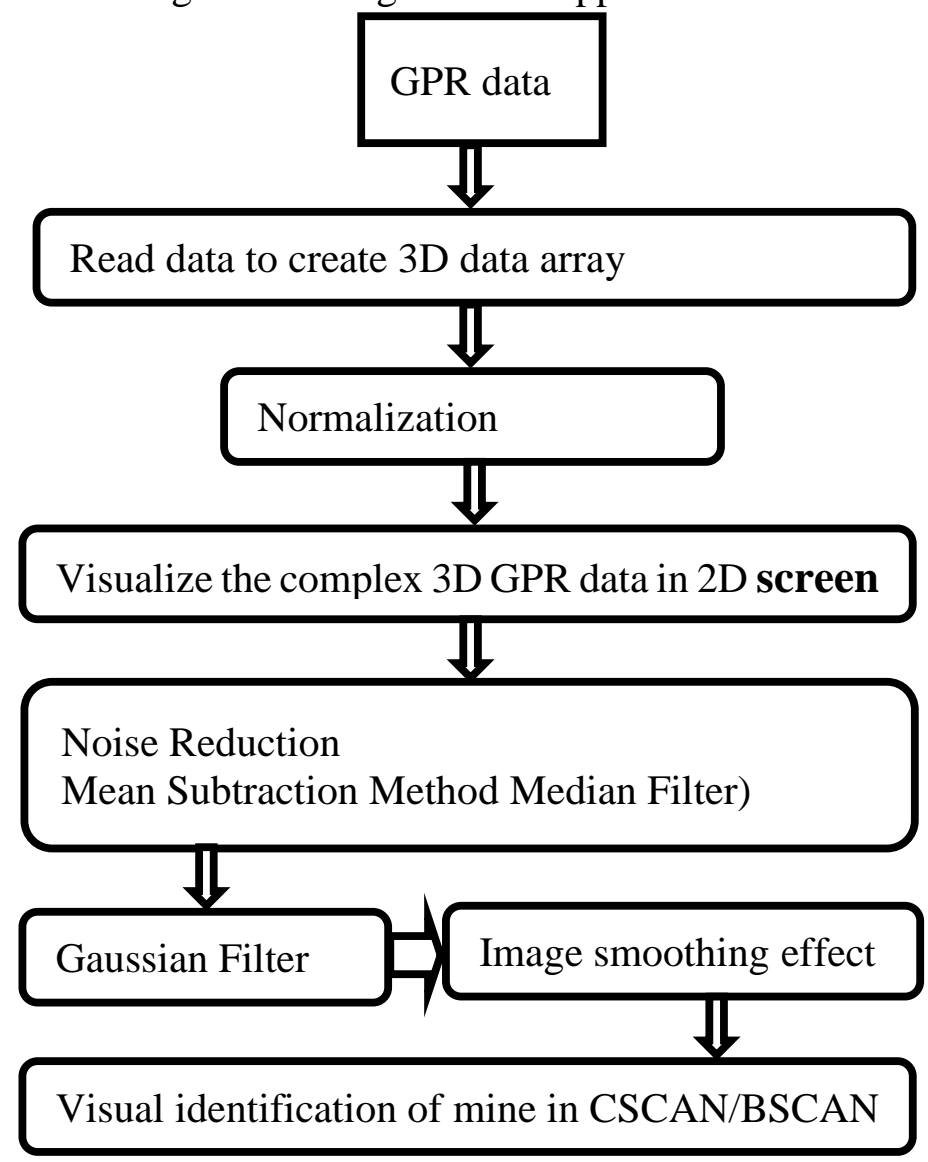

Fig. (2) GPR data normalizing procedure

\subsection{Multi-Aperture Processing for Improved Target Detection in Forward- Looking GPR Applications}

Standoff sensing with forward-looking ground-penetrating radar (FL-GPR) has been shown to be a promising technique to detect and localize heterogeneous buried targets [6]. The use of oblique and near-grazing incidence sensing configurations provide an FL-GPR the advantage of having a reduced return from the air-ground interface relative to its downward looking counterpart; it also permits more flexible operative conditions in comparison with groundcoupled sensors. However, a shortcoming of an FL-GPR system is that it suffers a reduction in the overall backscattered power from the illuminated buried targets, rendering the target returns comparable in strength to the clutter generated by the rough ground surface. Consequently, it is challenging to discern the target signature in the presence of these unwanted contributions, especially when the buried targets are non-metallic. As such, design and optimization of both imaging and detection procedures are essential to improved performance of the forward-looking modality. Recent works have proposed various methods to image and detect shallow target in forward looking modality. 


\subsection{Hidden Markov Models for Buried Objects Detection}

The Hidden Markov Models (HMM) are stochastic models for complex, nonstationary doubly stochastic processes that produce time sequences of random observations as a function of states. They are the most successful and widely used technique for speech recognition and are also very popular in handwriting recognition [7]. They have also been used in other applications to object recognition, such as target recognition. In speech recognition, an observation vector is extracted at each time over an interval of speech. The observation sequence is then processed by the HMM to produce probabilities that certain words generated the sequence. GPR systems also produce sequences of observation vectors that can be considered as functions of time. In hand-held systems, time-based sampling is typical. In vehicle mounted systems, sampling is usually performed as a function of position which is roughly equivalent to time, assuming constant velocity. As a vehicle with a GPR moves down a road, a multi-dimensional signal is produced at regular intervals. The signals from landmines can be modeled using HMMs to produce probabilities that sequences of these signals were produced by landmines and to infer the location of possible landmines.

\subsection{The FDTD Technique for Buried Objects Detection}

Maxwell's equations that are the governing equations of the GPR forward problem can be numerically solved using a variety of methods, among them the finite element method, the method of moments, implicit finite-difference techniques (Crank-Nicolson method, alternativedirection implicit), transmission line matrix, and others. The finite difference time-domain (FDTD) method is considered to be a very attractive choice for a number of reasons, the most important of which are its computational efficiency and its time domain nature that particularly suits the GPR problem [8]. Numerical modeling of GPR is considered to be an alternative interpretation approach and has been extensively applied to a number of GPR applications, among them are: the detection of dense nonaqueous phase liquids (DNAPL), the detection of geological targets like faults and caves, for tunnel inspections, detecting and assessing pipes, in the inspection and condition assessment of bridges, for forensic applications, mineral exploration, and airborne GPR. In the case of GPR numerical modeling for landmine detection, generic types of antennas over simple targets buried in both homogenous and inhomogeneous soils have been modeled. More advanced and realistic models for both antennas and targets are employed in order to simulate single GPR traces (A-Scans), which were subsequently used as a reference in an attempt to discriminate between landmines and false alarm targets.

\subsection{Dual Band SAR processing for IED Detection}

Detection and imaging of targets such as Improvised Explosive Devices (IEDs) in any given region of ground is possible because their constitutive dielectric parameters are different from anything else underground, but challenging because of the low dielectric contrast, unknown variation of the ground surface and volumetric inhomogeneities. Among other GPR techniques, Underground Focused as an appealing modality to solve the problem of finding buried threats as it is able to sense large areas of terrain in a short amount of time. This technology presents some challenges because it requires the estimation of the ground constitutive parameters that are necessary for the underground focusing process [9]. In addition, unknown variation of the ground surface and subsurface inhomogeneities obscure the target signal. The dual band implementation of the UFSAR algorithm is divided in two steps. First, the rough surface of the soil is estimated using the higher frequency band. Then, the rough soil estimation is used to both focus the underground spot and remove the surface clutter when calculating the UFSAR image in the lower band. This facilitates significant reduction of the rough surface clutter, improving the detection capabilities of the proposed system over those of previous approaches. 


\subsection{Classification of Buried Objects by using Genetic Programming and Neural Networks}

UXO classification using GPR technology often involves complex qualitative features and 2-D scattering images and is performed subjectively by human operators. Thus, inconsistent and subjective classification performance associated with human factors, such as fatigue, memory fading, learning capability, and feature complexity are clearly inevitable. In order to overcome this issue, an automatic, objective classification method without a trained operator is essential. Artificial intelligence (AI) techniques, such as neural networks (NN), have been investigated and applied with some success for developing autonomous classification algorithms for handwriting recognition, fault detection, medical diagnosis, as well as for buried UXO and landmine detection [10]. However, neural networks do suffer from some drawbacks. The network size and structure are user-defined, and there is no clear optimal architecture in a given application. Recently, genetic programming (GP), a relatively new AI method has been applied for classification. In the previous studies, GP was shown to be successfully applied to character recognition, biology, finance, and medicine classification problems. Genetic programming is an evolutionary learning method that is similar to the genetic algorithm (GA) and uses Darwinian natural selection to evolve computer programs to perform a given task. The main difference between GA and GP is that GA evolves and outputs a string of values or a solution vector, while GP evolves and outputs trees in the form of computer programs. Because GA works with solution vectors, the size and structure of the solution is restricted to the user-defined constraint. However, in GP, the size and structure of the solution are evolved to optimize the solution and are thus not limited. GP is similar to GA in its ability to search out the global optimal solution because it models the human invention process.

\subsection{Clutter Reduction Techniques for Detecting Subsurface Explosive Objects}

The outlines of this processing are shown in figure (3). The raw GPR data is input to preprocessing block. Preprocessing techniques are elementary signal processing methods applied to simplify the interpretation of the GPR data. Output of this block is processed to reduce/remove clutter using advanced signal processing techniques to filter out the ground reflection and effects of antenna of the GPR return data to enhance landmine detection. Minelike objects, such as stones and metal debris can also produce significant reflections which can be detected as alarms. The Wigner-Ville distribution (WVD) and the time frequency approach gives relevant feature extraction, Hidden Markov models (HMMs), the spectral feature vectors and subsurface detector, which are based on energy spectrum are also used as feature extraction of GPR signals. The purpose of these methods is trying to find the "hidden pattern" in the GPR return data. These features are used to distinguish landmines from mine-like objects.

\section{Pre-processing techniques}

Preprocessing techniques are elementary signal processing methods applied to simplify the interpretation of the GPR data. The main purpose of the preprocessors is for signal conditioning and data correction. These techniques include DC-offset removal (Dewowing), time-zero correction, noise reduction and antenna crosstalk removal.

\section{Clutter reduction techniques}

Out of four signal components in returned signal of GPR data, the noise and antenna cross talk can be reduced/removed by proper signal processing algorithms. These techniques include Antenna crosstalk, Noise Reduction and Air-Ground interface clutter. 


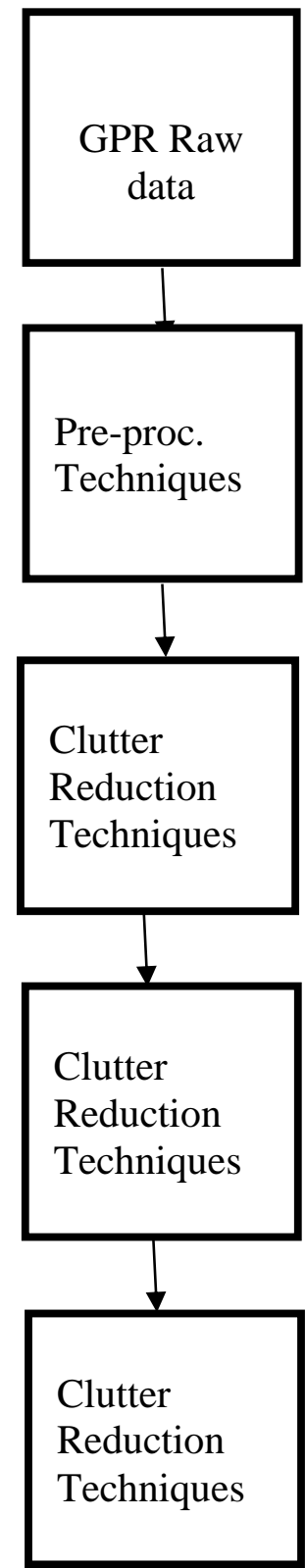

Fig. (3) Clutter Reduction Techniques Outlines

\section{Advanced signal processing techniques}

These techniques include Likelihood ratio test, Singular Value Decomposition (SVD), Principal Component Analysis (PCA), Independent Component Analysis (ICA), Wavelet Transform (WT), Kalman filter and Parameter Estimation.

\subsection{GPR Images Analysis using Neural Networks and Curve Fitting}

A brief outline of the methodology for the problem at hand is shown in figure (4). The objective is to detect, locate and identify a buried object in GPR image. Before undergoing the detection process, the images are first subjected to enhancement of its visualization using preprocessing technique. 


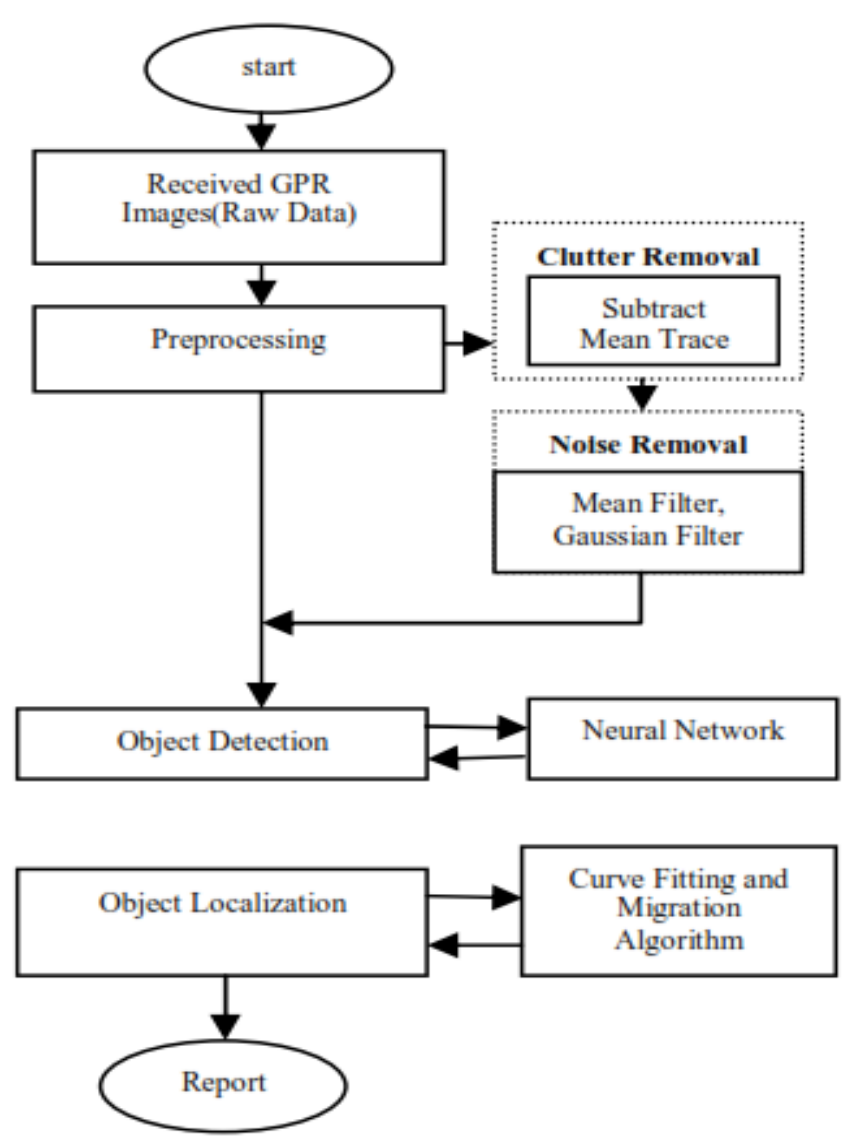

Fig. (4) Outline of Problem Methodology

\section{Conclusion}

In this paper, different modern signal processing techniques that are used recently, have been presented. In each section, a brief description of the techniques has been introduced to clarify the main processing steps and also to clarify the main advantages. The ongoing research is based on implementing one of these techniques to construct 3D images in the presence of strong unwanted signals, whether the target is with weak or strong reflection coefficient.

\section{References}

[1] Gülay Büyükaksoy Kaplan, Ahmet Burak Yoldemir, Oğuz İçoğlu, Mehmet Sezgin,"Discrimination of Buried Objects Using Angular Radial Transform and Multi-layer Perceptrons", 12th International Radar Symposium (IRS), 2011

[2] Hyoung-sun Youn, Chi-Chih Chen, "Neural Detection for buried piper using FullyPolarimetric Ground Penetrating Radar system", Antennas and Propagation Society International Symposium, 2003. IEEE

[3] Yue Yu; Chi-Chih Chen; Xuan Feng; Cai Liu, " Application of entropy classification method to the detection of subsurface linnear targets in polarimetric GPR data", 2016 IEEE International Geoscience and Remote Sensing Symposium (IGARSS).

[4] Ivor L. Morrow, Sebastian Wirth, Mark Finnis, "Discrimination of Buried Objects using Time-Frequency Analysis and Waveform Norms", Antennas \& Propagation Conference (LAPC), 2016 Loughborough. 
[5] v. G. Sugak, A. v. Bukin, N. G. Reznichenko, Ali Djadooei ,"Forward Looking Ground Penetrating Radar with Synthetic Antenna Aperture for Buried Explosive Hazards Detection", 21-24 June 2016, Kharkiv, Ukraine.

[6] D. Comite; F. Ahmad; M. G. Amin; T. Dogaru, "Multi-Aperture Processing for Improved Target Detection in Forward-Looking GPR Applications", 2016 10th European Conference on Antennas and Propagation (EuCAP), June 2016.

[7] P.D. Gader, M. MystkowskiYunxin Zhao, "Landmine Detection with Ground Penetrating Radar Using Hidden Markov Models", IEEE Transactions on Geoscience and Remote Sensing, Volume: 39, Issue: 6, Jun 2001.

[8] Iraklis Giannakis, Antonios Giannopoulos, Craig Warren, "A Realistic FDTD Numerical Modeling Framework of Ground Penetrating Radar for Landmine Detection", IEEE Journal of Selected Topics in Applied Earth Observations and Remote Sensing, Volume: 9, Issue: 1, Jan. 2016.

[9] Borja Gonzalez-Valdes ; Jose A. Martinez-Lorenzo ; Carey M. Rappaport, "Dual Band SAR Processing for Low Dielectric Contrast Buried IED Detection", July 2013.

[10]Classification of Buried Targets Using Ground Penetrating Radar: Comparison Between Genetic Programming and Neural Networks", IEEE Antennas and Wireless Propagation Letters, Volume 10, 2011. 